\title{
MicroRNA-101 suppresses colorectal cancer progression by negative regulation of Rap1b
}

\author{
ZHIYUAN ZHOU, HANG XU, YANTAO DUAN and BIN LIU \\ Department of General Surgery, Shanghai Ninth People's Hospital, \\ Shanghai Jiaotong University School of Medicine, Shanghai 200011, P.R. China
}

Received December 11, 2018; Accepted November 7, 2019

DOI: $10.3892 / \mathrm{ol} .2020 .11791$

\begin{abstract}
Colorectal cancer (CRC) is the fourth most lethal malignancy and is the second most common cause of cancer-associated mortality worldwide. The development of high-throughput sequencing has enabled the identification of potential biomarkers for the diagnosis and treatment of various types of cancer. Although microRNA-101 (miR-101) has been demonstrated to be a potential biomarker of CRC, its detailed mechanisms remain to be fully discovered. In the present study, overall survival analysis was applied to determine the association between miR-101 and CRC prognosis. Reverse transcription-quantitative PCR (RT-qPCR) was used to examine gene expression levels in tissues and cells. Cell proliferative and apoptotic activities were determined by MTT and flow cytometry assays, respectively. Wound healing and Transwell assays were used to examine CRC cell migration and invasion, respectively. In the present study, RT-qPCR analysis indicated that miR-101 was significantly downregulated in CRC tissues and cells. However, clinical data collected from The Cancer Genome Atlas revealed no significant association between the expression levels of miR-101 and the prognosis of CRC. Additionally, miR-101 inhibited the progression of CRC by directly binding to the 3'-untranslated region of Ras-related protein Raplb (Raplb). This was associated with downregulation of Rap1b expression. Furthermore, the overexpression of Raplb promoted miR-101 mimic-attenuated CRC cell progression. The present study demonstrated that miR-101 may be involved in the repression of the CRC progression by forming a negative feedback loop with Raplb. The findings revealed the interaction between miR-101 and Raplb during the progression of $\mathrm{CRC}$, which could aid the development of therapeutic strategies.
\end{abstract}

Correspondence to: Dr Bin Liu, Department of General Surgery, Shanghai Ninth People's Hospital, Shanghai Jiaotong University School of Medicine, 639 Manufacturing Bureau Road, Shanghai 200011, P.R. China

E-mail: bin_liu86@163.com

Key words: colorectal cancer, tumorigenesis and progression, microRNA-101, Ras-related protein Raplb

\section{Introduction}

According to the GLOBOCAN 2018 statistics, colorectal cancer (CRC) is the fourth (6.1\%) most common malignancy after lung (11.6\%), breast (11.6\%) and prostate cancer (7.1\%), whereas the mortality rate of CRC is the second highest $(9.2 \%)$ in the world, following lung cancer (18.4\%) (1). A total of 1.8 million CRC cases were diagnosed, and nearly 880,000 cases of CRC-associated mortality occurred in 2018 worldwide (1). An improvement in the treatment options of CRC is a significant challenge that requires attention. Additionally, the prioritization of primary prevention and early detection is also required.

MicroRNAs (miRNAs/miRs) belong to a class of short non-coding RNAs that are involved in cancer progression by binding to the complementary site of mRNAs, which often leads to translational repression or mRNA degradation (2). Various miRNAs, including let-7, miR-34a and the miR-200 family, have been identified as potential biomarkers or therapeutic molecules (3). Previous studies have reported that miR-101 is downregulated in various types of cancer and suppresses cancer cell progression, such as bladder cancer (4), breast cancer (5), hepatocellular carcinoma (6) and CRC (7). However, the comprehensive mechanisms underlying the effects of miR-101 on CRC progression have not been fully elucidated.

Ras-associated protein-1 (Rapl) serves a vital role in the regulation of cancer progression (8). This gene is a member of the Ras-like small GTPase family and contains two highly homologous isoforms, Rapla and Ras-related protein Raplb (Rap1b) (9). Several studies have proposed potential roles of Raplb in tumorigenesis and demonstrated the upregulation of Raplb in several types of cancer $(10,11)$. A previous study reported that the loss of Rap1b can attenuate thyroid cancer (12). Tang et al (13) indicated that Raplb promotes the invasion and migration of hepatocellular carcinoma cells by regulating Twist 1 . Jia et al (14) discovered that Rap1b can promote the progression of esophageal squamous cell carcinoma. Furthermore, Li et al (15) reported that metastasis associated lung adenocarcinoma transcript 1 can promote the proliferation of glioma cells by acting as a sponge for miR-101. In hepatocellular carcinoma, Sheng et al (16) reported that miR-101 regulates the progression of hepatocellular carcinoma by interacting with Raplb. Based on these findings and 
assumptions, it was hypothesized that miR-101 may interact with Raplb to suppress the progression of CRC.

The present study demonstrated that miR-101 inhibited CRC progression via regulation of Raplb. The present study provided insights into the underlying mechanism of miR-101 in CRC and facilitates development of therapeutic strategies for CRC.

\section{Materials and methods}

Clinical specimens. The tissues were collected from 50 patients (31 males and 19 females) with a median age of 48 years (range, 29-78 years) between April 2015 and June 2018 from Shanghai Ninth People's Hospital (Shanghai, China). Written informed consent was obtained from all patients prior to the study start. CRC tumor tissues were obtained via surgical resections of 50 patients, and adjacent normal tissues were obtained from the distal edge of each resection $<10 \mathrm{~cm}$ away from the tumor. The inclusion criteria for the present study were as follows, the patients were newly diagnosed and agreed to a 5-year follow up period. The exclusion criteria were as follows, patients diagnosed with other diseases and patients that had received chemotherapy or radiotherapy prior to surgery. All tissue samples were obtained and immediately stored at $-80^{\circ} \mathrm{C}$ prior to further experiments. The present study was approved by the Ethics Committee of Shanghai Ninth People's Hospital (Shanghai, China).

Cell lines. Human CRC cells (SW620), human colon mucosal cells (NCM460) and 293T cells were obtained from American Type Culture Collection. The cell lines were cultured in RPMI-1640 medium and supplemented with $10 \%$ fetal bovine serum. The 293T cells were cultured in Dulbecco's modified Eagle's medium (Gibco; Thermo Fisher Scientific, Inc.) with $10 \%$ fetal bovine serum (FBS; Thermo Fisher Scientific, Inc.) and $1 \%$ penicillin-streptomycin (Thermo Fisher Scientific, Inc). All cell lines were maintained at $37^{\circ} \mathrm{C}$ in a humidified atmosphere with $5 \% \mathrm{CO}_{2}$.

Reverse transcription-quantitative $(R T-q) P C R$. Total RNA was extracted from CRC tissues, adjacent normal tissues, SW620 cells and NCM460 cells using TRIzol ${ }^{\circledR}$ reagent (Invitrogen; Thermo Fisher Scientific, Inc.), and was reverse transcribed into cDNA by using a PrimeScript RT reagent kit (Takara Bio, Inc.) at $37^{\circ} \mathrm{C}$ for $15 \mathrm{~min}$, according to the manufacturer's protocol. The expression levels of miR-101 and Raplb were determined using a ViiATM 7 Real-Time PCR system (Thermo Fisher Scientific, Inc.). qPCR was subsequently performed using the SYBR ${ }^{\circledR}$-Green Real-Time PCR Master mix (Thermo Fisher Scientific, Inc.), according to the manufacturer's protocol. The following thermocycling conditions were used to detect miR-101: An initial predenaturation step at $50^{\circ} \mathrm{C}$ for $2 \mathrm{~min}$, followed by 40 cycles of denaturation at $95^{\circ} \mathrm{C}$ for $10 \mathrm{~min}$ and annealing at $60^{\circ} \mathrm{C}$ for $1 \mathrm{~min}$. For other factor detection, the thermocycling conditions were as follows: An initial predenaturation step at $94^{\circ} \mathrm{C}$ for $5 \mathrm{~min}$, followed by 40 cycles of denaturation at $95^{\circ} \mathrm{C}$ for $30 \mathrm{sec}$, annealing at $60^{\circ} \mathrm{C}$ for $30 \mathrm{sec}$ and extension at $72^{\circ} \mathrm{C}$ for $20 \mathrm{sec}$. The following primer sequences were used for the qPCR: Raplb; forward, 5'-TTT ATTCCATCACAGCACAGTCC-3' and reverse, 5'-TTTCTG
TTAATTTGCCGCACTAGG-3'; miR-101; forward, 5'-CGG CGGTACAGTACTGTGATAA-3' and reverse, 5'-CTGGTG TCGTGGAGTCGGCAATTC-3'; GAPDH; forward, 5'-GAT GATCTTGAGGCTGTTGTC-3' and reverse, 5'-CAGGGC TGCTTTTAACTCTG-3' and U6; forward, 5'-CTCGCTTCG GCAGCACATATACTA-3' and reverse, 5'-ACGAATTTG CGTGTCATCCTTGCG-3'. Relative expression levels were quantified using the $2^{-\Delta \Delta \mathrm{Cq}}$ method (17) and normalized to the internal reference genes GAPDH and U6.

Western blot analysis. Western blot analysis was performed in order to detect Raplb expression as previously described by Joseph et al (18). Total protein was extracted from transfected SW620 cells using radioimmunoprecipitation assay buffer (Beyotime Institute of Biotechnology). Total protein was quantified using a bicinchoninic acid assay and $10 \mu \mathrm{g}$ protein/lane was separated via SDS-PAGE on a $10 \%$ gel.Proteins were subsequently transferred onto polyvinylidene difluoride membrane and blocked in 5\% non-fat milk for $2 \mathrm{~h}$ at room temperature. The membranes were incubated with the following primary antibodies, anti-Raplb (1:1,000, cat. no. 2326; Cell Signaling Technology, Inc.) and anti-GAPDH (1:1,000, cat. no. 5174; Cell Signaling Technology, Inc.) overnight at $4^{\circ} \mathrm{C}$. Membranes were washed three times with TBS with $0.1 \%$ Tween-20. Following the primary incubation, membranes were incubated with horseradish peroxidase-labeled secondary antibodies $\left(1: 1,000\right.$, cat. no. ab205718; Abcam) for $2 \mathrm{~h}$ at $37^{\circ} \mathrm{C}$. Protein bands were visualized using SuperSignal ${ }^{\mathrm{TM}}$ West Femto Maximum Sensitivity Substrate (Thermo Fisher Scientific, Inc.), according to the manufacturer's protocol. GAPDH was used as an internal control.

Transfection. miR-101 mimics, anti-miR-101 and their respective negative controls (miRNA mimic/inhibitor) were synthesized by Shanghai GenePharma Co., Ltd. The full length of Rap1b was subcloned into pcDNA3.1 to overexpress Rap1b, with empty pcDNA3.1 vector serving as the control. Transfection of the cells with the miR-101 mimics $(10 \mathrm{nM})$, anti-miR-101 (10 nM), miRNA mimic/inhibitor (10 $\mathrm{nM})$ and vectors $(10 \mathrm{nM})$ were performed using Lipofectamine ${ }^{\circledR} 2000$ (Thermo Fisher Scientific, Inc.). Co-transfection of the cells with miR-101 mimic and Raplb was performed using Lipofectamine $^{\circledR} 2000$ (Thermo Fisher Scientific, Inc.), the subsequent experiments were performed at $48 \mathrm{~h}$ posttransfection. The sequences of oligonucleotides used were as follows: miR-101 mimics, 5'-UACAGUACUGUGAUA ACUGAA-3'; miRNA mimics, 5'-UUUGUACUACACAAA AGUACUG-3'; anti-miR-101, 5'-UUCAGUUAUCACAGU ACUGUA-3' and miRNA inhibitor control, 5'-UCACAACCU CCUAGAAAGAGUAGA-3'.

Cell proliferation, migration and invasion assays. An MTT assay was used to determine cell proliferation, whereas wound healing and Transwell assays were conducted to measure cell migratory and invasive activities, respectively. All of these assays were performed as previously described (16).

Flow cytometry. Cell apoptosis was detected in three independent experiments with the Annexin V-FITC/Propidium Iodide Apoptosis Detection kit (40302ES20), according to 
A



B

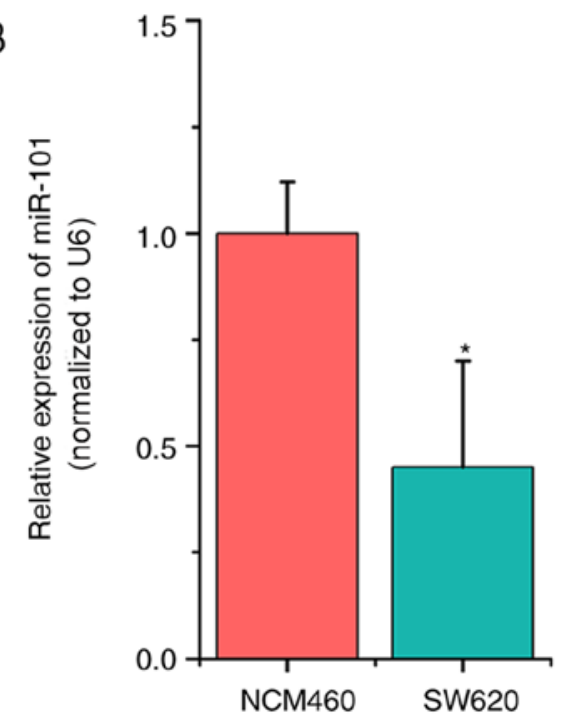

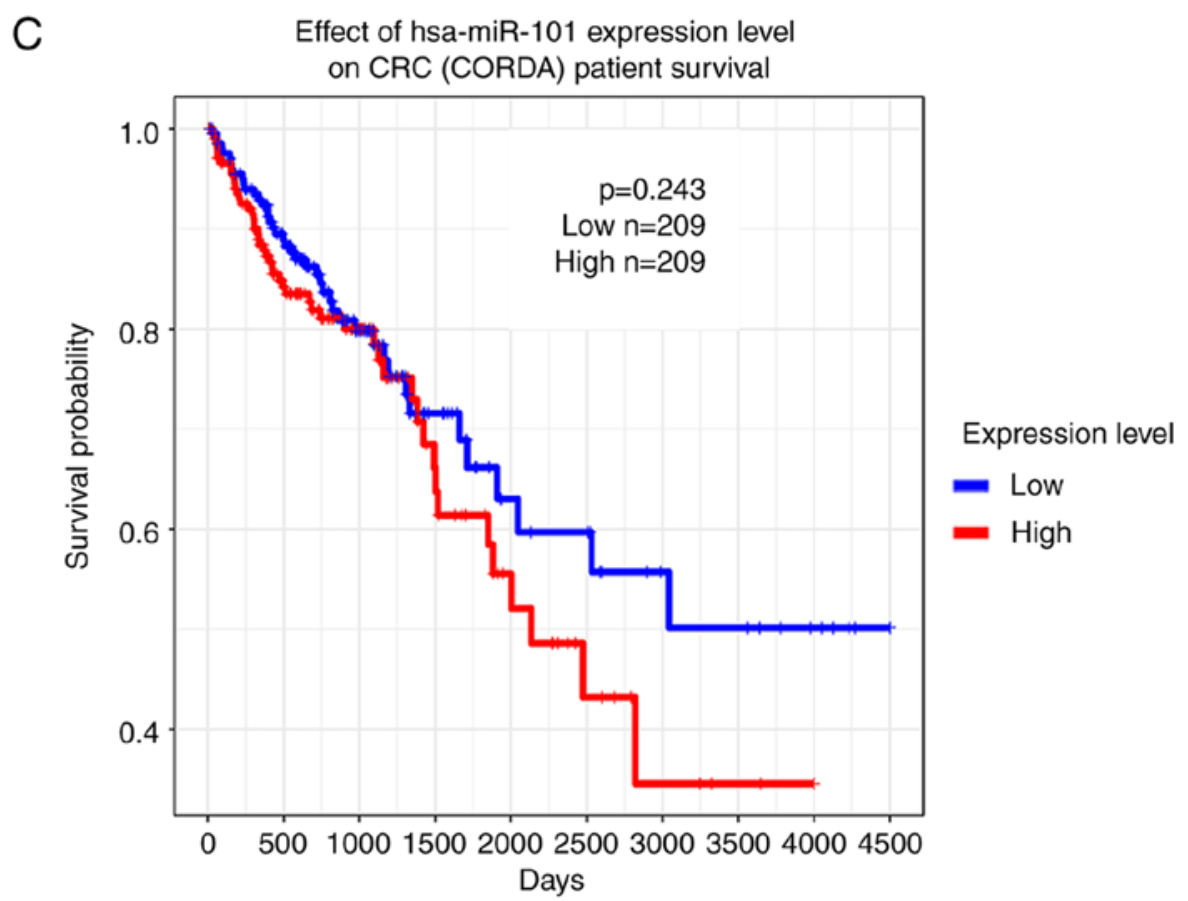

Figure 1. miR-101 expression is downregulated in CRC tissues and cells. (A) miR-101 expression was examined via RT-qPCR in CRC tissues and adjacent normal tissues $(\mathrm{n}=50)$. (B) miR-101 expression was examined via RT-qPCR in the CRC SW620 cell line and the normal human colon mucosal NCM460 cell line. (C) Association between miR-101 expression and CRC prognosis. " $\mathrm{P}<0.05$ vs. normal tissues; " $\mathrm{P}<0.05$ vs. NCMA60. CRC, colorectal cancer; RT-qPCR, reverse transcription-quantitative PCR; miR-101, microRNA-101; CORDA, colon adenocarcinoma.

the manufacturer's protocol. Cells were subsequently detected using the FACSCalibur flow cytometer (BD Biosciences) and analyzed using FlowJo software (version 7.6.1; FlowJo LLC). The apoptotic rate was presented as the percentage of Annexin V-positive cells among the total cells.

Luciferase reporter assay. 293 T cells were transfected with the Firefly luciferase plasmid (Promega Corporation) containing wild-type or mutant 3'-untranslated region (UTR) of Rap1b, using Lipofectamine ${ }^{\circledR} 2000$ (Thermo Fisher Scientific, Inc.). The cells were subsequently transfected with $\mathrm{NC}$, miR-101 mimic or anti-miR-101 (all from Ambion; Thermo Fisher Scientific, Inc.). After 48 h of cell culture, the cells were harvested and lysed. Luciferase activity was measured using the the Dual-Luciferase Reporter Analysis kit (Promega Corporation). Normalized relative light units represent Firefly luciferase activity/Renilla luciferase activity.

The Cancer Genome Atlas (TCGA) analysis. The clinical information and miR-101 expression profile of the CRC cases were obtained from TCGA database (https://cancergenome.nih.gov), within the colorecteral cancer dataset (TCGA-COAD) (19), using the R software package (version 3.1.3) (20). The expression levels of each hub gene were defined as either high (expression value $\geq$ median value) or low (expression value $<$ median value). The survival curves 

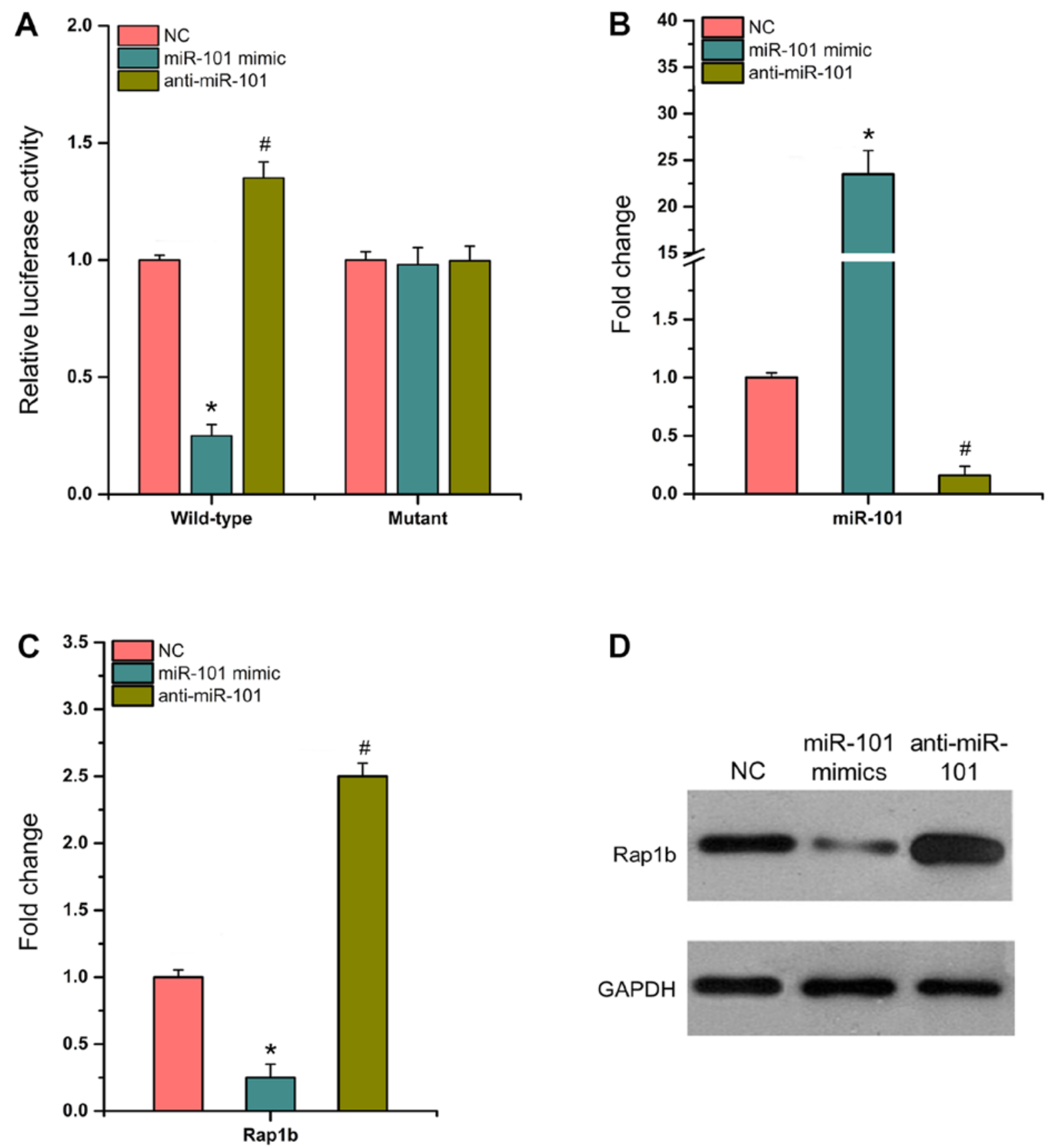

D



GAPDH

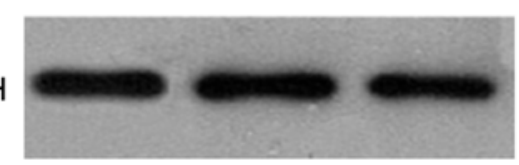

Figure 2. miR-101 directly targets Raplb and inhibits Raplb expression. (A) Dual luciferase reporter assays revealed luciferase activities in wild-type or mutant 3'-UTR-containing SW620 cells transfected with NC, miR-101 mimic or anti-miR-101.(B) RT-qPCR revealed the relative expression levels of miR-101 in SW620 cells transfected with NC, miR-101 mimic or anti-miR-101. (C) RT-qPCR revealed RAP1b mRNA expression in SW620 cells transfected with NC, miR-101 mimic or anti-miR-101. (D) Western blotting revealed the Raplb protein expression in SW620 cells transfected with NC, miR-101 mimic or anti-miR-101. GAPDH was used as the internal control. ${ }^{*} \mathrm{P}<0.05,{ }^{\#} \mathrm{P}<0.05$ vs. NC. Rap1b, Ras-related protein Rap1b; NC, negative control; miR-101, microR NA-101; RT-qPCR, reverse tanscription-quantitative PCR.

were analyzed via the Kaplan-Meier method, and univariate survival analysis was performed using a log-rank test.

Statistical analysis. The software package SPSS (version 22.0; IBM Corp.) was used for statistical analysis. The data derived from each experiment were presented as the mean \pm standard deviation. A paired Student's t-test was used to compare parameters between two groups, and one-way ANOVA and Tukey's test were used to evaluate the differences among multiple groups. $\mathrm{P}<0.05$ was considered to indicate a statistically significant difference. The experiments were performed $>3$ times.

\section{Results}

miR-101 expression is downregulated in CRC tissues and cells. In order to explore the detailed role of miR-101 in CRC, 50 paired tumor tissues and their adjacent normal tissues were collected. Subsequently, the expression levels of miR-101 were detected. RT-qPCR results indicated that miR-101 expression was significantly lower in CRC tumor tissues compared with in normal tissues $(\mathrm{P}<0.05$; Fig. $1 \mathrm{~A}$;). The present study further examined miR-101 expression in CRC cells (SW620) and human colon mucosal cells (NCM460), which indicated similar results 
A



D

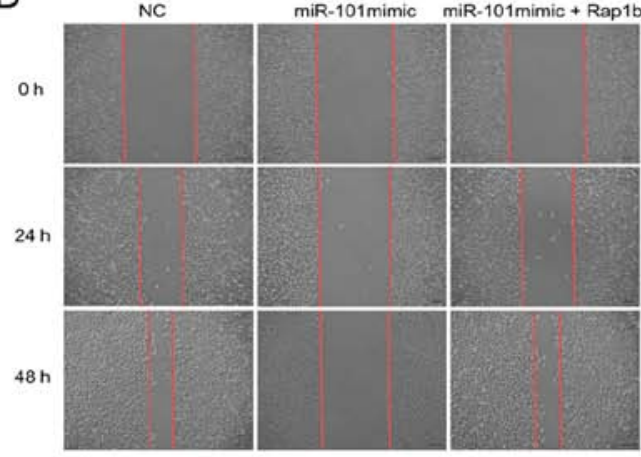

$B$

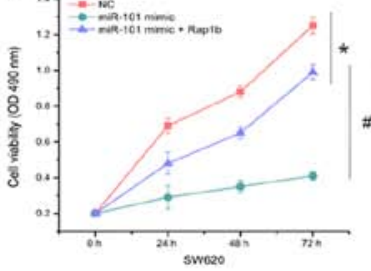

C
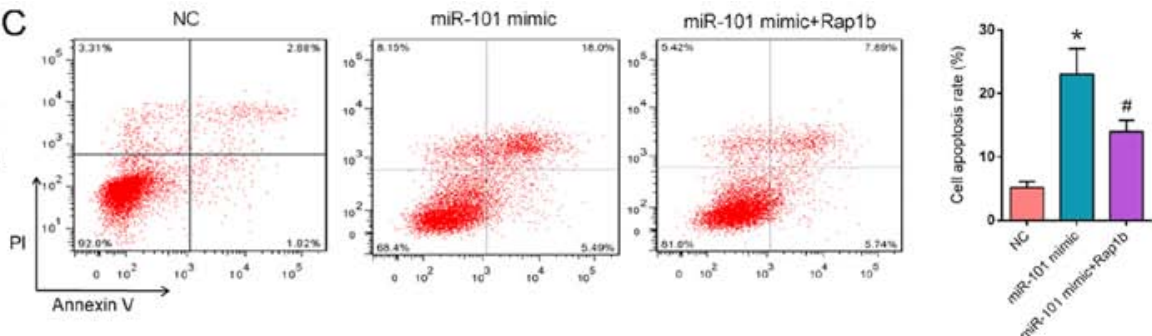

E



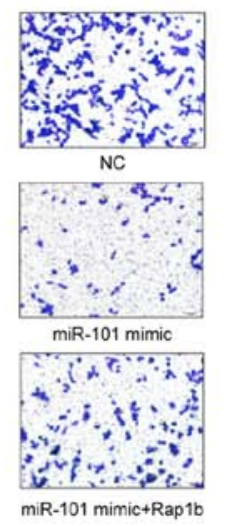

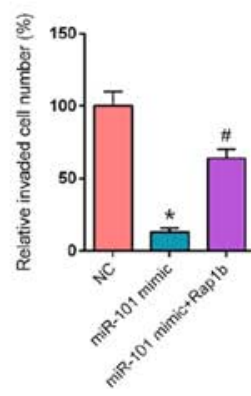

Figure 3. Overexpression of Raplb rescues miR-101 mimic-attenuated CRC progression. (A) RT-qPCR revealed the relative Raplb expression in SW620 cells transfected with pcDNA3.1 and pcDNA3.1- Rap1b. (B) MTT assays revealed the cell proliferation rate of SW620 cells transfected with NC, miR-101 mimic or miR-101 mimic + Raplb at different time points $(0,24,48$ and $72 \mathrm{~h}$ ). (C) Flow cytometry revealed cell apoptosis of SW620 cells transfected with NC, miR-101 mimic or miR-101 mimic + Raplb. (D) Wound healing assays revealed migration abilities of SW620 cells transfected with NC, miR-101 mimic or miR-101 mimic + Raplb at 0,24 and 48 h. (E) Transwell invasion assays demonstrated invasion abilities of SW620 cells transfected with NC, miR-101 mimic or miR-101 mimic + Raplb. " $\mathrm{P}<0.05$ vs. NC; ${ }^{\prime} \mathrm{P}<0.05$ vs. miR-101 mimic. Raplb, Ras-related protein Raplb; NC, negative control; miR-101, microRNA-101; PI, propidium iodide; RT-qPCR, reverse transcription-quantitative PCR.

( $\mathrm{P}<0.05$; Fig. 1B). In addition, the association between miR-101 expression and the prognosis of patients with CRC was investigated using TCGA data. The overall survival curves indicated no apparent association between miR-101 expression levels and the prognosis of patients with $\mathrm{CRC}(\mathrm{P}=0.243$; Fig. 1C).

miR-101 directly targets Raplb and inhibits its expression. The present study used dual luciferase reporter assays to detect luciferase activity in 293T cells containing wild-type or mutant 3'-UTR, which were transfected with anti-miR-101, miR-101 mimic and NC. The results indicated that in wild-type SW620 cells, miR-101 mimic significantly decreased luciferase activity by $\sim 75 \%$ compared with that noted in NC cells, whereas anti-miR-101 significantly enhanced luciferase activity by $\sim 40 \%$ ( $\mathrm{P}<0.05$; Fig. 2A). The transfection efficiency of miR-101 mimic and anti-miR-101 in SW620 cells was confirmed ( $\mathrm{P}<0.05$; Fig. 2B). RT-qPCR and western blotting assays demonstrated that the expression levels of Raplb were significantly decreased by miR-101 mimic (Fig. 2C and D; $\mathrm{P}<0.05)$. Overall, the data indicated that miR-101 could directly bind to Raplb and downregulate its expression in CRC cells.

miR-101 inhibits the development and progression of CRC cells. MTT and flow cytometry assays indicated that miR-101 overexpression could significantly inhibit cell proliferation and promote apoptosis (Fig. 3A and B). Wound healing assays further demonstrated that miR-101 mimic decreased SW620 cell migration (Fig. 3C). Transwell assays indicated that miR-101 mimic suppressed SW620 cell invasion (Fig. 3D). Overall, the results indicate that miR-101 acts as a tumor suppressor for CRC.
Overexpression of Raplb rescues miR-101 mimic-attenuated CRC progression. In order to further determine whether the interaction between miR-101 and Raplb could affect the progression of CRC, Raplb was transfected into miR-101 mimic-expressing SW620 cells and the transfection efficiency of Raplb was measured. The expression of Raplb significantly increased in SW620 cells transfected with pcDNA3.1-Raplb compared with SW620 cells transfected with pcDNA3.1 ( $\mathrm{P}<0.05$; Fig. 3A). Furthermore, MTT assays demonstrated that Raplb overexpression significantly enhanced miR-101-suppressed cell proliferation $(\mathrm{P}<0.05$; Fig. 3B). Flow cytometry assays indicated that aberrantly expressed Raplb significantly decreased the apoptosis rate induced by miR-101 mimic $(\mathrm{P}<0.05 ;$ Fig. $3 \mathrm{C})$. In addition, Raplb reversed the suppressive effect of miR-101 on cell migration and invasion in SW620 cells $(\mathrm{P}<0.05$; Fig. 3D and $\mathrm{E}$, respectively). In summary, the results of the present study suggest that Raplb is a key downstream effector of miR-101-regulated CRC phenotypes.

\section{Discussion}

Abnormal expression of miRNAs is a feature of human malignancies and frequently leads to either oncogenesis or tumor suppression $(21,22)$. Increasing evidence has demonstrated that miR-101 is downregulated in various types of cancer and inhibits the development and progression of cancer $(23,24)$. Liu et al $(25)$ reported that miR-101 can interact with cancer susceptibility $2 \mathrm{c}$ to promote astrocy toma tumorigenesis. Furthermore, Jiang et al (26) demonstrated that miR-101 is involved in CRC metastasis via the miR-101-O-linked $\beta$-N-acetylglucosamine/enhancer of zeste 
2 polycomb repressive complex 2 subunit regulatory feedback circuit. Xiong et al (27) revealed that CRC cell proliferation and migration are regulated by the zinc finger E-box binding homeobox 1 (ZEB1)-transcription factor AS1/miR-101/ZEB1 axis. The present study determined that miR-101 was significantly downregulated in CRC tissues and cells according to RT-qPCR assays. Additionally, SW620 cells transfected with miR-101 mimic exhibited decreased growth rates compared with NC cells. In addition, it was demonstrated that the upregulation of miR-101 promoted cell apoptosis. Wound healing and Transwell assays demonstrated that miR-101 could suppress CRC cell migration and invasion.

Previous studies have demonstrated that Raplb is involved in the progression of various types of cancer and that it often acts as an oncogene to promote cancer cell proliferation (28-30). Peng et al (31) highlighted that miR-100 can regulate $\mathrm{CRC}$ cell proliferation and invasion by targeting Raplb, and Guo et al (32) demonstrated that miR-139 acts as a sponge of Raplb to affect the progression of CRC, which indicated that Raplb may regulate CRC progression through multiple mechanisms. Therefore, it was inferred that miR-101 could bind to the complementary sequence of Raplb and may affect the biological functions of CRC cells. Initially, a dual luciferase reporter assay was applied to confirm the interaction between miR-101 and Raplb. miR-101 mimic significantly downregulated Raplb expression in wild-type SW620 cells. Furthermore, overexpression of Rap1b could rescue miR-101 mimic-attenuated cell growth, migration and invasion.

Collectively, the present study identified a molecular axis of miR-101/Raplb underlying CRC progression. However, in vivo experiments are required to further validate these findings in follow-up studies. It remains necessary to identify downstream signaling pathways that are regulated by Raplb.

\section{Acknowledgements}

Not applicable.

\section{Funding}

No funding was received.

\section{Availability of data and materials}

The datasets used and/or analyzed during the present study are available from the corresponding author on reasonable request.

\section{Authors' contributions}

$\mathrm{ZZ}$ and BL designed the study. $\mathrm{HX}$ and $\mathrm{YD}$ analyzed the data and prepared the figures. $\mathrm{ZZ}$ and $\mathrm{BL}$ drafted the initial manuscript. All authors read and approved the final manuscript.

\section{Ethics approval and consent to participate}

The present study was approved by the Ethics Committee of the Shanghai Ninth People's Hospital (Shanghai, China). Written informed consent was obtained from all patients prior to the study start.

\section{Patient consent for publication}

Not applicable.

\section{Competing interests}

The authors declare that they have no competing interests.

\section{References}

1. Bray F, Ferlay J, Soerjomataram I, Siegel RL, Torre LA and Jemal A: Global cancer statistics 2018: GLOBOCAN estimates of incidence and mortality worldwide for 36 cancers in 185 countries. CA Cancer J Clin 68: 394-424, 2018.

2. Bartel DP: MicroRNAs: Genomics, biogenesis, mechanism, and function. Cell 116: 281-297, 2004.

3. Rupaimoole R and Slack FJ: MicroRNA therapeutics: Towards a new era for the management of cancer and other diseases. Nat Rev Drug Discov 16: 203-222, 2017.

4. Liu D, Li Y, Luo G, Xiao X, Tao D, Wu X, Wang M, Huang C, Wang L, Zeng F and Jiang G: LncRNA SPRY4-IT1 sponges miR-101-3p to promote proliferation and metastasis of bladder cancer cells through up-regulating EZH2. Cancer Lett 388: 281-291, 2017.

5. Qian K, Liu G, Tang Z, Hu Y, Fang Y, Chen Z and Xu X: The long non-coding RNA NEAT1 interacted with miR-101 modulates breast cancer growth by targeting EZH2. Arch Biochem Biophys 615: 1-9, 2017.

6. Liu Y, Tan J, Ou S, Chen J and Chen L: MicroRNA-101-3p suppresses proliferation and migration in hepatocellular carcinoma by targeting the $\mathrm{HGF} / \mathrm{c}-\mathrm{Met}$ pathway. Invest New Drugs: Mar 30, 2019 (Epub ahead of print). doi: 10.1007/s10637019-00766-8.

7. Chen LG, Xia YJ and Cui Y: Upregulation of miR-101 enhances the cytotoxic effect of anticancer drugs through inhibition of colon cancer cell proliferation. Oncol Rep 38: 100-108, 2017.

8. Hattori M and Minato N: Rap1 GTPase: Functions, regulation, and malignancy. J Biochem 134: 479-484, 2003.

9. Pei W, Tao L, Zhang LW, Zhang S, Cao J, Jiao Y, Tong J and Nie J: Circular RNA profiles in mouse lung tissue induced by radon. Environ Health Prev Med 22: 36, 2017.

10. Yang Y, Li M, Yan Y, Zhang J, Sun K, Qu JK, Wang JS and Duan XY: Expression of RAP1B is associated with poor prognosis and promotes an aggressive phenotype in gastric cancer. Oncol Rep 34: 2385-2394, 2015.

11. Lin KT, Yeh YM, Chuang CM, Yang SY, Chang JW, Sun SP, Wang YS, Chao KC and Wang LH: Glucocorticoids mediate induction of microRNA-708 to suppress ovarian cancer metastasis through targeting Rap1B. Nat Commun 6: 5917, 2015.

12. Huk DJ, Ashtekar A, Magner A, La Perle K and Kirschner LS: Deletion of Raplb, but not Rapla or Epac1, reduces protein kinase A-mediated thyroid cancer. Thyroid 28: 1153-1161, 2018.

13. Tang Z, Peng H, Chen J, Liu Y, Yan S, Yu G, Chen Q, Tang H and Liu S: Raplb enhances the invasion and migration of hepatocellular carcinoma cells by up-regulating Twist 1 . Exp Cell Res 367: 56-64, 2018.

14. Jia Z, Yang Y, Dengyan Z, Chunyang Z, Donglei L, Kai W and Song Z: RAP1B, a DVL2 binding protein, activates Wnt/beta-catenin signaling in esophageal squamous cell carcinoma. Gene 611: 15-20, 2017

15. Li Z, Xu C, Ding B, Gao M, Wei X and Ji N: Long non-coding RNA MALAT1 promotes proliferation and suppresses apoptosis of glioma cells through derepressing Rap1B by sponging miR-101. J Neurooncol 134: 19-28, 2017.

16. Sheng Y, Ding S, Chen K, Chen J, Wang S, Zou C, Zhang J, Cao Y, Huang A and Tang H: Functional analysis of miR-101-3p and Rap1b involved in hepatitis B virus-related hepatocellular carcinoma pathogenesis. Biochem Cell Biol 92: 152-162, 2014.

17. Livak KJ and Schmittgen TD: Analysis of relative gene expression data using real-time quantitative PCR and the 2(-Delta Delta C(T)) method. Methods 25, 402-408, 2001.

18. Joseph JV, Conroy S, Pavlov K, Sontakke P, Tomar T, Eggens-Meijer E, Balasubramaniyan V, Wagemakers M, den Dunnen WF and Kruyt FA: Hypoxia enhances migration and invasion in glioblastoma by promoting a mesenchymal shift mediated by the HIF1 $\alpha$-ZEB1 axis. Cancer Lett 359: 107-116, 2015. 
19. Collins FS and Barker AD: Mapping the cancer genome Pinpointing the genes involved in cancer will help chart a new course across the complex landscape of human malignancies. Sci Am 296: 50-57, 2007.

20. Li L, Zhu Z, Zhao Y, Zhang Q, Wu X, Miao B, Cao J and Fei S: FN1, SPARC, and SERPINE1 are highly expressed and significantly related to a poor prognosis of gastric adenocarcinoma revealed by microarray and bioinformatics. Sci Rep 9: 7827, 2019.

21. Hassanlou M, Soltani BM, Medlej A, Kay M and Mowla SJ: Hsa-miR-6165 downregulates insulin-like growth factor-1 receptor (IGF-1R) expression and enhances apoptosis in SW480 cells. Biol Chem: Nov 8, 2019 (Epub ahead of print). doi: 10.1515/hsz-2018-0421.

22. Zhu QL, Zhan DM, Chong YK, Ding L and Yang YG: MiR-652-3p promotes bladder cancer migration and invasion by targeting KCNN3. Eur Rev Med Pharmacol Sci 23: 8806-8812, 2019.

23. Cao C, Xu Y, Du K, Mi C, Yang C, Xiang L, Xie Y and Liu W: LINC01303 functions as a competing endogenous RNA to regulate EZH2 expression by sponging miR-101-3p in gastric cancer. J Cell Mol Med 23: 7342-7348, 2019.

24. Zhang H, Wang X, Hu B, Zhang F, Wei H and Li L: Circular RNA ZFR accelerates non-small cell lung cancer progression by acting as a miR-101-3p sponge to enhance CUL4B expression. Artif Cells Nanomed Biotechnol 47: 3410-3416, 2019.

25. Liu C, Sun Y, She X, Tu C, Cheng X, Wang L, Yu Z, Li P, Liu Q, Yang $\mathrm{H}$, et al: CASC2c as an unfavorable prognosis factor interacts with miR-101 to mediate astrocytoma tumorigenesis. Cell Death Dis 8: e2639, 2017.

26. Jiang M, Xu B, Li X, Shang Y, Chu Y, Wang W, Chen D, Wu N, $\mathrm{Hu}$ S, Zhang S. et al: O-GlcNAcylation promotes colorectal cancer metastasis via the miR-101-O-GlcNAc/EZH2 regulatory feedback circuit. Oncogene 38: 301-316, 2019.
27. Xiong WC, Han N, Wu N, Zhao KL, Han C, Wang HX, Ping GF, Zheng PF, Feng H, Qin L and He P: Interplay between long noncoding RNA ZEB1-AS1 and miR-101/ZEB1 axis regulates proliferation and migration of colorectal cancer cells. Am J Transl Res 10: 605-617, 2018.

28. Wilson JM, Lorimer E, Tyburski MD and Williams CL: $\beta$-Adrenergic receptors suppress Rap1B prenylation and promote the metastatic phenotype in breast cancer cells. Cancer Biol Ther 16: 1364-1374, 2015.

29. Zhang M, Zhou S, Zhang L, Zhang J, Cai H, Zhu J, Huang C and Wang J: miR-518b is down-regulated, and involved in cell proliferation and invasion by targeting Raplb in esophageal squamous cell carcinoma. FEBS Lett 586, 3508-3521, 2012

30. Li Y, Liu Y, Shi F, Cheng L and She J: Knockdown of Rap1b enhances apoptosis and autophagy in gastric cancer cells via the PI3K/Akt/mTOR pathway. Oncol Res 24: 287-293, 2016.

31. Peng H, Luo J, Hao H, Hu J, Xie SK, Ren D and Rao B: MicroRNA-100 regulates SW620 colorectal cancer cell proliferation and invasion by targeting RAP1B. Oncol Rep 31: 2055-2062, 2014.

32. Guo H, Hu X, Ge S, Qian G and Zhang J: Regulation of RAP1B by miR-139 suppresses human colorectal carcinoma cell proliferation. Int J Biochem Cell Biol 44: 1465-1472, 2012.

This work is licensed under a Creative Commons Attribution-NonCommercial-NoDerivatives 4.0 International (CC BY-NC-ND 4.0) License. 